\title{
Modelling and Analysis of Effect of Awareness Programs by Media on the Spread of COVID-19 Pandemic Disease
}

\author{
Fekadu Tadege Kobe \\ Department of Mathematics, College of Natural and Computational Science, Wachemo University, Hossana, Ethiopia
}

Email address:

feket200@gmail.com

\section{To cite this article:}

Fekadu Tadege Kobe. Modelling and Analysis of Effect of Awareness Programs by Media on the Spread of COVID-19 Pandemic Disease. Mathematics and Computer Science. Vol. 5, No. 5, 2020, pp. 93-102. doi: 10.11648/j.mcs.20200505.12

Received: October 21, 2020; Accepted: November 4, 2020; Published: December 11, 2020

\begin{abstract}
This paper proposes and analyses a basic deterministic mathematical model to investigate Modeling and Analysis of effect of awareness program by media on the spread COVID-19 Pandemic Disease. The model has seven non-linear differential equations, which describe the effects of awareness programs by media on the spread of COVID-19 Pandemic diseases. Analytical study carried out to investigate the model analysis and existence of stability of system, given threshold parameters known as the basic reproduction number, which obtained using next generation matrix method. The equilibrium of COVID 19 models is determined. In addition to having a disease-free equilibrium, which is globally asymptotically stable when the basic reproduction number less than one, COVID 19 model manifest one's possession of (a quality of) the phenomenon of backward bifurcation where a stable disease-free equilibrium co-exists (at the same time) with a stable endemic equilibrium for a certain range of associated reproduction number less than one. The analysis and simulation results of the model suggested that the most effective strategies for controlling or eradicating the spread of COVID 19 pandemic were suggest using that awareness programs through the media campaigning are helpful in decreasing the spread of COVID 19 Pandemic diseases by isolating a fraction of susceptible from infective.
\end{abstract}

Keywords: COVID-19 Pandemic, Awareness Programs, Stability Analysis, SEIR Model and Reproduction Number

\section{Introduction}

Coronaviruses are a large family of viruses that may cause illness in humans that known to cause respiratory infections ranging from the common cold to more severe diseases such as Middle East Respiratory Syndrome (MERS) and Severe Acute Respiratory Syndrome (SARS). In today's world, many countries have faced with a global threat whose source is not precisely yet known: A novel coronavirus. In December 2019, a series of pneumonia cases unknown cause emerged in Wuhan, with clinical presentation greatly resembling viral pneumonia and has rapidly spread too many countries in the world and has seriously shook up humanity.

On 30 January 2020, the WHO Emergency Committee declared a global health emergency based on growing case notification rates at Chinese and international locations. The case detection rate is changing daily and can tracked in almost real-time on the website provided by Johns Hopkins University [1] and other forums. As of midst of February 2020, China bears the large burden of morbidity and mortality, because the incidence in other Asian countries, in Europe and North America remains low so far. Coronaviruses are envelopes, positive single-stranded large RNA viruses that infect humans, but also a range of animals. Tyrrell and Byron, who cultivated the viruses from patients with common colds [2], first described Coronaviruses in 1966. Based on their morphology as spherical versions with a core shell and surface projections resembling a solar corona, they were termed coronaviruses (Latin: corona $=$ crown). Four subfamilies, namely alpha-, beta-, gamma- and delta corona viruses exist. While alpha- and beta-coronaviruses apparently originate from mammals, in particular from bats, gammaand delta-viruses originate from pigs and birds.

Among the seven subtypes of coronaviruses that can infect humans, the beta-coronaviruses may cause severe disease and fatalities, whereas alpha-coronaviruses cause asymptomatic or symptomatic infections. SARSCoV-2 belongs to the B line age of the beta-coronaviruses and closely related to the SARS-CoV [3, 4]. The major four structural genes encode the nucleocapsid protein $(\mathrm{N})$, the spike protein $(\mathrm{S})$, a small 
membrane protein (SM) and the membrane glycoprotein (M) with an additional membrane glycoprotein (HE) occurring in the HCoV-OC43 and HKU1 beta-coronaviruses [5]. Symptoms COVID 19 and symptomatic infections especially affect young children [6]. Observations so far suggest a mean incubation period of five days [7] and a median incubation period of 3 days (range: 0-24 days). The proportion of individuals infected by SARS-CoV-2 who remain asymptomatic throughout the course of infection had not yet definitely assessed. In symptomatic patients, the clinical manifestations of the disease usually start after less than a week, consisting of fever, cough, nasal congestion, fatigue and other signs of upper respiratory tract infections. The infection can progress to severe disease with dyspnea and severe chest symptoms corresponding to pneumonia in approximately $75 \%$ of patients, as seen by computed tomography on admission [8]. Pneumonia mostly occurs in the second or third week of a symptomatic infection. Prominent signs of viral pneumonia include decreased oxygen saturation, blood gas deviations, changes visible through chest X-rays and other imaging techniques, with ground glass abnormalities, patchy consolidation, alveolar exudates and interlobular involvement, eventually indicating deterioration. Lymphopenia appears to be common, and inflammatory markers (C-reactive protein and proinflammatory cytokines) are elevated. Since, it had spread to 198 countries and territories around the world and had declared a pandemic. As of March 31, 2020, there have been reported 750,874 covid-19 cases, 36,045 deaths, and 117,603 recoveries [9]. The first confirmed COVID-19 case in Africa reported in Egypt on February 14, 2020 and since then the number of confirmed infections in the region kept increasing and has surpassed over 5000 cases by March 31, 2020 [10, 11]. Comoros, Lesotho, Malawi, and South Sudan are the only African countries that had not reported a confirmed case by March 31, 2020.

Ethiopia, being one of the countries with limited trained human and material resources, expected to affect most by the global COVID-19 pandemic. Allocating the limited resources for the prevention of transmission of the disease and implementation of a uniform and evidence based preventive and treatment protocol at all levels of health care system and throughout the country under central command believed to be wise decision for optimal utilization of the resources. Areas affected most given priority to treat and contain the infection in that locality so that other part of the country not affected. In order to make all preventive and treatment endeavors uniform in Ethiopia the need for national COVID-19 prevention and treatment guideline given priority by $\mathrm{FMOH}$ and EPHI. A committee organized from consultants of different specialties and given the task of developing evidence based, cost effective and applicable national guideline for prevention and treatment of COVID-19 in Ethiopia. The guideline has taken in to consideration the culture, leaving condition and background of the people and is make as much as possible understandable and usable by most levels of health care professional
This prevention and treatment guideline encompasses principles of infection prevention and control, starting from the scene up to discharge and safe burial system in case of death. Management of critically ill patients, in wards and ICU had discussed in the guideline. Ethical considerations regarding safety of health professionals and bioethics have been included. WHO and CDC base the recommendations in this guideline on limited studies available and recommendation. The guideline revised and updated as more information and evidences released. All healthcare facilities must ensure that health professionals are well trained and able to implement infection control procedures and COVID19 management [12].

On 13 March 2020, Ethiopia confirmed its first case of COVID-19. Ethiopia, with a population of over 105 million people, is a migration hotspot for regional movements, through Africa and to the Gulf. It is home to over 750,000 refugees from neighbouring countries and is a hub of trade. It has one of the world's largest internally displaced populations, primarily from conflicts. The country has a uniquely complex mobility environment and a regional governance system. At present the spread of COVID-19, the regional governments have taken individual measures to close and control their borders with neighbouring regions while restricting internal movements. Federal reforms had met with increased local tensions in many communities, which have sought to solidify ethnic majorities and marginalize minorities ahead of now postponed 2020 elections. The first case was registered in Ethiopia march 15, 2020 imported cases only and currently the FMOH has reported May 23, 2020 Covid-19 laboratory conformed case 4,663 , death 75 and recovered 1,295. These relatively low numbers could be due to limited availability of COVID-19 test kits, lack of community testing (those tested come through airport screening), and undetected people with no or mild symptoms not seeking care and thus not tested (represent a significant proportion of infections). Thus, this number may represent the tip of the iceberg so the rate of transmissions more from import case and some case was made with personal contract were reported [13].

To date, no vaccine is available for COVID-19. Therefore, the ability to limit the devastating consequences of the disease on people's lives and livelihoods would have to rely on the implementation of effective preventative nonpharmaceutical interventions (NPIs). NPIs are multiple public health measures designed to reduce viral transmission rates in a population by reducing the reproduction number $\left(R_{0}\right)$; the average number of secondary cases each case generates $[14,15]$. NPIs directly influence the course of the COVID-19 pandemic, including the rate of spread, and the expected duration of the pandemic. However, characteristics of the virus such as incubation period (the time between infection and symptom onset), serial interval (the time between symptom onset of a primary and secondary case) the extent of asymptomatic cases, the possibility of pre symptomatic infectiousness, and the case fatality rate (CFR). In addition, the possible role of weather in transmission, all 
remain ambiguous or mostly unknown [16, 17]. Asymptomatic or mild presentation comprises the bulk of the reported cases, which is an estimated at $80 \%$ [18]. With the lack of clinical studies measuring vermeil, the infectious period also remains largely unknown, with estimates ranging from few days to 10 days or more after the incubation period [19].

The complexity of the infection and recovery process, therefore, means that proper understanding of the epidemiological dynamics of COVID-19 within the local context is fundamental to combat the pandemic. Specifically, studies illustrating future trajectories of the disease can help nations and communities to develop early warning systems, avoid overwhelming healthcare services, minimize morbidity and mortality from the disease, and evaluate the effects of interventions and the long-term consequences of the virus on peoples' livelihood. This is particularly true in Africa, where livelihoods are fragile and previous epidemics, such as HIV/AIDS and, more recently, Ebola had known to exert enormous socioeconomic consequences [14, 15]. In addition, in the face of a new pandemic in the region, the already overstretched healthcare systems that are struggling to deliver essential healthcare services such as immunization and HIV/AIDS treatment would be in further jeopardy and risk losing the gains achieved so far in disease control efforts.

In fact, there are many imminent questions about the spread of COVID-19. How many people will be infected tomorrow? When will the inflection point of the infection rate appear? How many people will be infected during the peak period? Can existing interventions effectively control the COVID-19? What mathematical models are available to help us answer these questions? The COVID-19 is a novel coronavirus that only discovered in December 2019, so data on the outbreak is still insufficient, and medical means such as clinical trials are still in a difficult exploratory stage [20]. So far, epidemic data have been difficult to apply directly to existing mathematical models, and questions need to be addressed as to how effective the existing emergency response has been and how to invest medical resources more scientifically in the future and so on. Based on this, this article aims to study the gaps in this part.

In the present study, we formulate and analyze an SEIR model to study the impact of awareness programs conducted by a media campaign on the spread of COVID 19 pandemic disease, in a variable population with immigration. In the modeling process, it assumed that the growth rate of the cumulative density of awareness programs driven by the media depends upon the number of invectives present in the population and extending the model SEIR by adding selfquarantine, highly awarded Infected, Unaware sever infected and infected diagnosed comportment will be considered [2627]. Further, the awareness about the disease will alert the susceptible to self-quarantine or stay at home and who will understand the symptoms isolate themselves from infective and avoid being infected by forming a separate class or infected diagnosed or hospitalized them. Take the effect of depletion of awareness programs with time have also into account while modeling and analyzing the system. The proposed model had visualized as the co-existence of causing a COVID 19 pandemic disease [27-28].

\section{Model Formulation and Analysis}

\subsection{Model Formulation}

The SEIR is a deterministic mathematical model and has four classes or compartments Susceptible, Exposed, Infected and Recovered. The effect of controlling technique in the spread of malaria, HIV/AIDS, TB, and COVID-19 is analyzed. Using these notations, seven classes of compartmental models are possible, SI, SEIR, SEI, SEIS, SIR, SIRS, SPITR, SEIR and SEIRS [26-29]. For example, in an SEIRS model, a fraction of the susceptible (S) population gets to infection, a part of which then becomes infectious (I). Some from the I class recover from the disease, and become part of the $\mathrm{R}$ class with temporary immunity. When immunity is lost, they become susceptible to pathogen attack again, and enter the $\mathrm{S}$ class. The simulation studies of the model with variable values of sensitive parameter of the spread of COVID-19 pandemic diseases are performed by extending the above models there were done such like new estimated model were done in mathematical modeling. For examples SEIQDR, $\mathrm{SE} I_{m} I_{s} I_{D} R$ and $\mathrm{SE} S_{q} I_{1} I_{2} R V$ model done in different country with mathematical modeling researchers in the COVID-19 epidemic diseases [29, 34].

\subsection{Model Assumption}

After the outbreak of the COVID-19 epidemic, the Chinese government has taken many effective measures to combat the epidemic, such as inspection detention, isolation treatment, isolation of cities, and stopping traffic on main roads $[22,23]$. However, the traditional SEIR model cannot fully describe the impact of these measures on different populations. Based on the analysis of the actual situation and existing data, we divided the population into different aware houses and established a more effective model for the dynamic spread of infectious diseases.

According to the actual situation of the pandemics, we divided the population into 7 different categories or compartement to comply with the current spread of COVID19 pandemic diseases in Ethiopia. See the Table 1 for specific classification. Since the incubation period of the COVID-19 is as long as 2 to 14 days, there are already infected but undetected people (E) in the natural environment of the susceptible population (S), when the first case is identified. Some people who have been infected need to go through a certain incubation period before suspected symptoms can be detected $\left(S_{q}\right)$. Chest CT imaging was used to observe whether there were glassy shadows in the lungs to determine whether the diagnosis was conformed $\left(I_{D}\right)$, unaware infected individuals with severe symptoms $\left(I_{s}\right)$, awareness fades Infected with the virus and highly awarded infectious $\left(I_{a}\right)$ and after a period of quarantine treatment, these two groups 
of people will be recovered from hospital (R), or face death due to basic diseases.

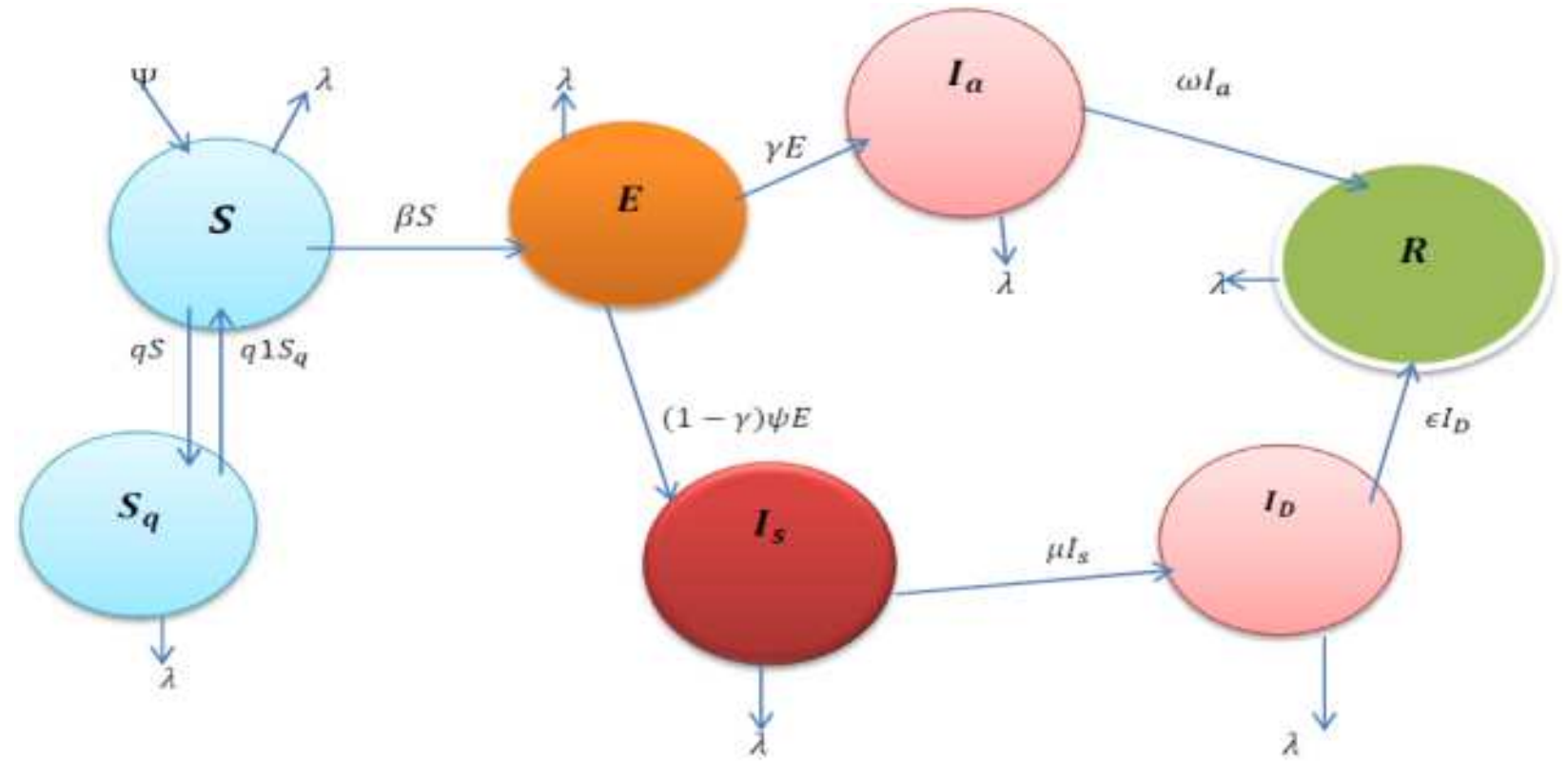

Figure 1. Schematic of the transmission COVID 19 model with disease-awareness by media $\left(S S_{q} E I_{a} I_{s} I_{D} R-m_{0}\right.$ del).

Table 1. Parameters and their interpretations for the COVID-19 model.

\begin{tabular}{ll}
\hline Parameter & Description \\
\hline$\beta$ & The incidence rate of susceptible population \\
$q$ & Transfer rate of humans from susceptible to self-quarantine susceptible \\
$q 1$ & Transfer rate of self-quarantine susceptible to susceptible after medical diagnosis \\
$\mu$ & Transfer rate of confirmed laboratory positive case \\
$\omega$ & Transfer rate of awarded cure or died to recovered \\
$\epsilon$ & Transfer rate of medical diagnosis cure or died to recovered \\
$\Psi$ & The recruitment rate at which new individuals enter in the Ethiopian population \\
$(1-\gamma) \psi$ & Reduction of exposed to severe infected \\
$\lambda$ & Natural death rate \\
$\gamma$ & Transfer of rate at the proportion of latent persons who were converted to free infection awarded humans \\
\hline
\end{tabular}

Table 2. Variables of the basic COVID-19 model.

\begin{tabular}{ll}
\hline Variables & Description \\
\hline$S(t)$ & People who may be infected by the virus at time $t$ \\
$E(t)$ & Infected with the virus but without the typical symptoms of infection at time $t$ \\
$I_{a}(t)$ & Infected with the virus and highly awarded infectious at time $t$ \\
$I_{S}(t)$ & Infected individuals with severe symptoms at time $t$ \\
$I_{D}(t)$ & Infected individuals Diagnosed and quarantined at time $t$ \\
$S_{q}(t)$ & Number of People self-quarantine susceptible at time $t$ \\
$R(t)$ & Number of people who are cured after infection at time $t$ \\
$N(t)$ & Total human population at time $t$ \\
\hline
\end{tabular}

Shown are epidemiological transitions in the baseline transmission model (black arrows). Susceptible persons $(S)$ become latently infected $(E)$ with the force of infection contact with infectious individuals in two infectious classes $\left(I_{a}\right.$ and $\left.I_{s}\right)$. Individuals leave the $E$ compartment at rate $\gamma \psi$. A proportion $\gamma$ of the latently infected individuals $(E)$ will go to the $I_{a}$ compartment, and the proportion $(1-\gamma)$ of $E$ individuals will go to the $I_{s}$ compartment. Infectious individuals with mild symptoms $\left(I_{a}\right)$ recovered at rate $\omega$. Individuals with severe symptoms $\left(I_{S}\right)$ are diagnosed and kept in isolation $\left(I_{D}\right)$ at rate $\epsilon$ they recover $(R)$ or die. Table 1 and 2 provides the description and values of all parameters. Applying the assumptions, definitions of compartmental variables and parameters described in tables 1 and 2, the system of non-linear differential equations, which describe the dynamics of COVID 19 pandemic disease transmission with help of making awareness by media are formulated and presented in this section.

$$
\begin{gathered}
\frac{d S}{d t}=\Psi-\frac{\beta S I_{a}}{N}-(\lambda+q) S+q 1 S_{q} \\
\frac{d E}{d t}=\frac{\beta S I_{a}}{N}-(\gamma+\lambda+\psi-\gamma \psi) E \\
\frac{d I_{a}}{d t}=\gamma \psi E-(\lambda+\omega) I_{a} \\
\frac{d I_{s}}{d t}=(1-\gamma) \psi E+(\lambda+\mu) I_{s} \\
\frac{d I_{D}}{d t}=\mu I_{s}-(\lambda+\epsilon) I_{D}
\end{gathered}
$$




$$
\begin{aligned}
& \frac{d R}{d t}=\omega I_{a}+\epsilon I_{D}-\lambda R \\
& \frac{d S_{q}}{d t}=q S-(\lambda+q 1) S_{q}
\end{aligned}
$$

With initial conditions:-

$S(0)>S 0, E(0) \geq E 0, S_{q}(0)>S_{q} 0, I_{a}(0) \geq$ $I_{a} 0, I_{s}(0) \geq I_{s} 0, I_{D}(0) \geq I_{D} 0, R(0)>R 0$. The total number of population size is $N=S+E+S_{q}+I_{a}+I_{s}+I_{D}+R$ with their differential.

\subsection{Model Analysis}

We analyze the model to check if the awareness by media has any influence on the diseases, that is, whether the disease eradicated or not. The thresholds parameters that determine persistence or elimination of COVID 19 will be determined and studied. Therefore, we start by determining the invariant region to check whether the model is biologically meaningful and showing that all solutions 0 of system equations are positive for all $t \geq 0$ and are attracted in that region.

\subsubsection{Non- Negativity of Solution}

Theorem 2.3.1 Each solution of the $S E S_{q} I_{a} I_{S} I_{D} R$ model structure (1) with preliminary stipulations subsists in the interval $[0, \infty)$ and $\mathrm{S}(\mathrm{t})>0, S_{q}(\mathrm{t}) \geq 0, \mathrm{E}(\mathrm{t}) \geq 0, I_{a}(\mathrm{t}) \geq 0, I_{s}(\mathrm{t})$ $\geq 0, I_{D}(\mathrm{t}) \geq 0$ as well as $\mathrm{R}(\mathrm{t})>0$ for all values of $\mathrm{t} \geq 0$.

Proof. As the right hand side of $S E S_{q} I_{a} I_{s} I_{D} R$ model structure (1) is completely continuous and locally LipSchitzian on $C$, the solution $\left(S(t), S_{q}(t), E(t), I_{a}(t), I_{s}(t)\right.$, $\left.I_{D}(t), R(t)\right)$ with initial conditions exists, and is unique on [0, $\zeta)$, where $0<\zeta<\infty$. From system (1) with initial condition, we have

$$
\frac{d s}{d t}>-A S(t), S(t) \geq S(0) \exp [-A t]>0
$$

The second equation of the system implies

$$
\frac{d E}{d t} \geq-B E(t), E(t) \geq E(0) \exp [-B t] \geq 0
$$

Again from the system equations we have

$$
\begin{gathered}
\frac{d S_{q}}{d t}>-C S_{q}(t), S_{q}(t) \geq S_{q}(0) \exp [-C t]>0 \\
\frac{d I_{a}}{d t} \geq-D I_{a}(t), I_{a}(t) \geq I_{a}(0) \exp [-D t] \geq 0 \\
\frac{d I_{s}}{d t} \geq-D I_{s}(t), I_{s}(t) \geq I_{s}(0) \exp [-E t] \geq 0
\end{gathered}
$$$$
\Omega=\left(\left(S, S_{q}, E, I_{a}, I_{S}, I_{D}, R\right) \in \mathbb{R}^{7+}: S>0, S_{q} \geq\right.
$$

Therefore, the region $\Omega$ is positively invariant (i.e solutions remain positive for all times, $t$ ) and in the model system equations (1-7) is biologically meaningful and mathematically well posed in the domain $\Omega$.

\subsubsection{Existence of Equilibrium Point}

Steady state solutions or equilibrium points are the roots or

$$
\begin{gathered}
\frac{d I_{D}}{d t} \geq-D I_{D}(t), I_{D}(t) \geq I_{D}(0) \exp [-F t] \geq 0 \\
\frac{d R}{d t}>-G R(t), R(t) \geq R(0) \exp [-G t]>0
\end{gathered}
$$

\subsubsection{Invariant Region}

The total population sizes are $N=S+S_{q}+E+I_{a}+I_{s}+$ $I_{D}+R$ with their differential equations.

$$
\frac{d N}{d t}=\frac{d S}{d t}+\frac{d S_{q}}{d t}+\frac{d E}{d t}+\frac{d I_{a}}{d t}+\frac{d I_{S}}{d t}+\frac{d I_{D}}{d t}+\frac{d R}{d t}=\Psi-\lambda N
$$

Theorem 2.3.2. The solutions of the system (1) are feasible for all $\mathrm{t} \geq 0$ if they enter the invariant region $\Omega$.

Proof. Let $\left(S, S_{q}, E, I_{a}, I_{s}, I_{D}, R\right) \in \mathbb{R}^{7+}$ be any solution of the system (1) with non-negative initial conditions.

$$
\frac{d N}{d t} \leq \Psi-\lambda N, \frac{d N}{d t}+\lambda N \leq \Psi
$$

(2) The integrating factor for (10) is $(I F)=e^{\int-\lambda d t}=$ $e^{-\lambda t}$ Multiplying both side of (10) by $e^{-\lambda t}$ gives

$$
e^{-\lambda t \frac{d N}{d t}+} \lambda N e^{-\lambda t} \leq \Psi e^{-\lambda t}
$$

integrating on both sides of (10) we have

$$
\lambda N e^{-\lambda t} \leq \frac{\Psi}{\lambda} e^{-\lambda t}+c
$$

Where $c$ is a constant of integration. Dividing through (17) by $e^{-\lambda t}$ gives

$$
\lambda N e^{-\lambda t} \leq \frac{\Psi}{\lambda} e^{-\lambda t}+c
$$

Using the initial conditions at $t=0, N_{0}=0$

$$
N_{0} \leq \frac{\Psi}{\lambda}+c \Rightarrow N_{0}-\frac{\Psi}{\lambda} \leq c
$$

Substitute in equation (13) we can get

$$
N \leq \frac{\Psi}{\lambda}+\left(N_{0}-\frac{\Psi}{\lambda}\right) e^{-\lambda t}
$$

Applying the theorem of differential inequality [30], we obtain.

$0 \leq N \leq \frac{\Psi}{\lambda}$ as $t \rightarrow \infty$. Therefore, as $t \rightarrow \infty$ in (20), the human population $N$, approaches $k=\frac{\Psi}{\lambda}$ (that is $N \rightarrow k=\frac{\Psi}{\lambda}$ ), the parameters $k=\frac{\Psi}{\lambda}$ usually called the carrying capacity [31].

Hence all feasible solutions set of the human population of the model (1) enters the region.

$\left.0, E \geq 0, I_{a} \geq 0, I_{s} \geq 0, I_{D} \geq 0 R>0, \mathrm{~N} \leq \frac{\Psi}{\lambda}\right)$

solutions of the system of equations when the right-hand side of a nonlinear system is set to zero. That is, using the nonlinear system (1) we have

$$
\begin{gathered}
\Psi-\frac{\beta S I_{a}}{N}-(\lambda+q) S+q 1 S_{q}=0 \\
\frac{\beta S I_{a}}{N}+(\gamma+\lambda+\psi-\gamma \psi) E=0
\end{gathered}
$$




$$
\begin{gathered}
\gamma \psi E-(\lambda+\omega) I_{a}=0 \\
(1-\gamma) \psi E+(\lambda+\mu) I_{s}=0 \\
\mu I_{s}-(\lambda+\epsilon) I_{D}=0 \\
\omega I_{a}+\epsilon I_{D}-\lambda R=0 \\
q S-(\lambda+q 1) S_{q}=0
\end{gathered}
$$

Let $\left(S^{*}, S^{*}{ }_{q}, E^{*}, I^{*}{ }_{a}, I^{*}{ }_{S}, I^{*}{ }_{D}, R^{*}\right)$ be the steady state of (17) which can obtain by solving (21-27). Disease free equilibrium points (DFE) are steady state solutions where there is no COVID 19 conformed in the population that is $S, S_{q}, E, I_{a}, I_{S}, I_{D}$ and $R$. In absence of the disease, this implies that $E=I_{a},=I_{s}=I_{D},=R=0$ therefore reduces to

$$
\left.\begin{array}{c}
\Psi-(\lambda+q) S=0 \\
q S-(\lambda+q 1) S_{q}=0
\end{array}\right\}
$$

This implies that

$$
\left.\begin{array}{c}
S^{*}=\frac{\Psi}{\lambda+q} \\
S^{*}{ }_{q}=\frac{q \Psi}{(\lambda+q)(\lambda+q 1)}
\end{array}\right\}
$$

Therefore, the disease-free equilibrium point of the COVID 19 model (1) is given by,

$$
\begin{gathered}
E_{0}=\left(S^{*}, S^{*}{ }_{q}, E^{*}, I^{*}{ }_{a}, I^{*}{ }_{S}, I^{*}{ }_{D}, R^{*}\right)= \\
\left(\frac{\Psi}{\lambda+q}, \frac{q \Psi}{(\lambda+q)(\lambda+q 1)}, 0,0,0,0,0,\right)
\end{gathered}
$$

This represents the state in which there is no infection (in the absence of COVID 19 cases) in the society.

\subsubsection{Reproduction Number}

Reproduction number, denoted by $R_{o}$, is the threshold or a level for many epidemiological models [21]. It determines whether a disease can attack the population or not. The threshold quantity $R_{0}$ indicates one infected individual produces the number of new infected individuals. When $R_{0}<0$ each infected individual propagates the infection and produces on average less than one new infected individual so that the disease is expected to die out completely over time. On the other hand if $R_{0}>1$, each individual produces more than one new infected individual so we would expect the disease to spread more and grow in the population. This means that the value of threshold quantity $R_{0}$ in order to eradicate the disease must reduce less than one. The following steps followed to compute the basic reproduction number $R_{0}$ the basic reproduction number cannot be determined from the structure of the mathematical model alone, but depends on the definition of infected and uninfected compartments. Assuming that there are $n$ compartments of which the first $m$ compartments to infected individuals. That is the parameters may be vary compartment to compartment, but are the identical for all individuals within a given compartment. Let

$$
X_{i}=\left(x_{1}, x_{2}, \ldots \ldots \ldots . . . x_{n}\right), X_{i} \geq 0 \text { for all, } i=1,2, \ldots \ldots . m
$$

Be the vector of human and mosquito individuals in each compartment. Let us sort the compartments so that first $m$ compartments infected individuals.

Let $F_{i}(x)$ be the rate of appearance of new infections in compartment $i$.

$V_{i}(x)=V^{-}{ }_{i}(x)-V^{+}{ }_{i}(x)$ Where $V^{+}{ }_{i}(x)$ is rate of transfer of individuals into compartment $i$ by all other means and $V^{-}{ }_{i}(x)$ is the rate of transfer of individual out of the $i^{t h}$ compartment.

It assumed that each function is continuously differentiable at least twice in each variable. The disease transmission model consists of non-negative initial conditions together with the following system of equations:

$$
\frac{d x_{i}}{d t}=f_{i}(x)=F_{i}(x)-V_{i}(x), i=1,2,3 \ldots . n
$$

Where $\frac{d x_{i}}{d t}$ is the rate of change. The next is the computation of the square matrices $F$ and $V$ of order ( $m x m)$, where $m$ is the number of infected classes, defined by $F=\left[\frac{d F_{i}(x)}{d x j}\left(x_{0)}\right]\right.$ and $V=\left[\frac{d V_{i}(x)}{d x i}\left(x_{0}\right)\right]$ with $1 \leq i, j \leq m$, such that $F$ is non- negative, $V$ is non-singular matrix and $x_{0}$ is the disease-free equilibrium point (DFE). Since $F$ is nonnegative and $V$ is non-singular, then $V^{-1}$ is non-negative and $F V^{-1}$ is non-negative. Hence the $F V^{-1}$ called the next generation matrix for the model. Finally, the basic reproduction number $R_{0}$ given by

$$
R_{0}=\gamma\left(F V^{-1}\right)
$$

Where $\gamma(A)$ denotes the spectral radius of matrix $A$ and and the spectral radius is the biggest non-negative eigenvalue of the next generation matrix. Rewriting model system (1) starting with the infected compartments for the population; $S, S_{q}, E, I_{a}, I_{S}, I_{D}, R$ and then following by uninfected classes; $S, S_{q}, R$, also from the population, then the model system becomes

$$
\left.\begin{array}{c}
\frac{d E}{d t}=\frac{\beta S I_{a}}{N}+(\gamma+\lambda+\psi-\gamma \psi) E \\
\frac{d I_{a}}{d t}=\gamma \psi E-(\lambda+\omega) I_{a} \\
\frac{d I_{s}}{d t}=(1-\gamma) \psi E+(\lambda+\mu) I_{S} \\
\frac{d I_{D}}{d t}=\mu I_{S}-(\lambda+\epsilon) I_{D} \\
\frac{d S}{d t}=\Psi-\frac{\beta S I_{a}}{N}-(\lambda+q) S+q 1 S_{q} \\
\frac{d S_{q}}{d t}=q S-(\lambda+q 1) S_{q} \\
\frac{d R}{d t}=\omega I_{a}+\epsilon I_{D}-\lambda R
\end{array}\right\}
$$

From the system of equation (19) $F_{i}$ and $V_{i}$ are defined as

$$
F(x)=\left[\begin{array}{c}
\frac{\beta S I_{a}}{N} \\
0 \\
0 \\
0
\end{array}\right] V(x)=\left[\begin{array}{c}
(\gamma+\lambda+\psi-\gamma \psi) E \\
\gamma \psi E-(\lambda+\omega) I_{a} \\
(1-\gamma) \psi E+(\lambda+\mu) I_{s} \\
\mu I_{s}-(\lambda+\epsilon) I_{D}
\end{array}\right]
$$

The partial derivatives of (19) with respect to $\left(I_{a}\right)$ and the jacobian matrix of $F_{i}$ at the disease-free equilibrium point 
(18) is:-

$$
F=\left[\begin{array}{llll}
0 & \beta & 0 & 0 \\
0 & 0 & 0 & 0 \\
0 & 0 & 0 & 0 \\
0 & 0 & 0 & 0
\end{array}\right]
$$

Similarly, the partial derivatives of (19) with respect to $\left(E, I_{a}, I_{S}, I_{D}\right)$ and the jacobian matrix $v$ is:-

$$
\begin{gathered}
v=\left[\begin{array}{cc}
(\gamma+\lambda+\psi-\gamma \psi) 00 & 0 \\
0-(\lambda+\omega) & 00 \\
(1-\gamma) \psi 0(\lambda+\mu) & 0 \\
00 \mu & -(\lambda+\epsilon)
\end{array}\right] \\
v^{-1}=\left[\begin{array}{ccc}
\frac{1}{(\gamma+\lambda+\psi-\gamma \psi)} 0 & 0 & 0 \\
0 \frac{1}{(\gamma+\lambda+\psi-\gamma \psi)} & 0 & 0 \\
\frac{1}{(1-\gamma) \psi} 0 & \frac{1}{\lambda+\mu} & 0 \\
00 \frac{\mu}{\lambda+\epsilon} \frac{1}{\lambda+\epsilon}
\end{array}\right]
\end{gathered}
$$

From $F v^{-1}$, we can determine the eigenvalues of the basic reproduction number $R_{0}$ by taking the spectral radius (dominant eigenvalue) of the matrix $F v^{-1}$. Thus it is calculated as $|A-\lambda A|=0$. We determine the expression for $R_{0}$ using the next generation matrix approach [22] as $R_{0}=\frac{\beta}{(\gamma+(1-\gamma) \psi+\lambda)}$. Further, it verified that the disease free equilibrium point $E_{0}$ given by (18) is locally asymptotically stable if $R_{0}<1$ and unstable if $R_{0}>1$.

\subsubsection{Stability of the Disease-Free Equilibrium}

The local stability of the disease-free equilibrium analyzed using the Jacobian matrix of the malaria model (1) at the disease free equilibrium point. Using [32], the following theorem contains.

Theorem 2.3.3 The disease free equilibrium point for system (1) is locally asymptotically stable if $R_{0}<1$ and unstable if $R_{0}>1$.

Proof. The Jacobian matrix $(J)$ of the malaria model (1) with $S=N-\left(E, I_{a}, I_{S}, I_{D}\right)$ and $S q=N-\left(E, I_{a}, I_{S}, I_{D}\right)$ at the disease-free equilibrium point is given by

$$
\left.\begin{array}{c}
\frac{d E}{d t}=\frac{\beta S I_{a}}{N}+(\gamma+\lambda+\psi-\gamma \psi) E \\
\frac{d I_{a}}{d t}=\gamma \psi E-(\lambda+\omega) I_{a} \\
\frac{d I_{s}}{d t}=(1-\gamma) \psi E+(\lambda+\mu) I_{S} \\
\frac{d I_{D}}{d t}=\mu I_{S}-(\lambda+\epsilon) I_{D}
\end{array}\right\}
$$

From the equation (20) the jacobian matrix

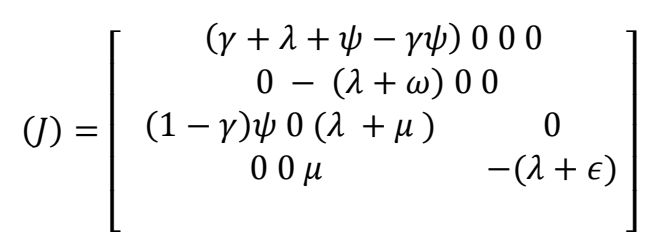

Let $\quad a=(\gamma+\lambda+\psi-\gamma \psi), b=(\lambda+\omega), c=$ $(\lambda+\mu), d=(\lambda+\epsilon), e=(1-\gamma) \psi$ and $f=\mu$

Thus

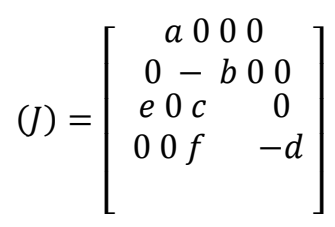

The eigenvalues of jacobian matrix are:-

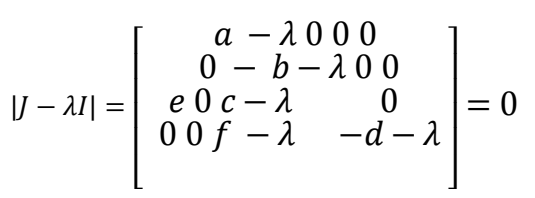

The second column has diagonal entry, therefore one of the eigenvalues of the jacobian matrix is $-(\lambda+\omega)$ or $b$. Applying Routh-Hurwitz stability criterion.

Here, we need find the eigenvalue of the system from the Jacobian matrix (32)

$$
a-\lambda\left|\begin{array}{cc}
c-\lambda & 0 \\
f-\lambda & -d-\lambda
\end{array}\right|
$$

We obtain the characteristic polynomial

$$
\lambda^{3}-(a+c-d) \lambda^{2}-(c a-a d+c d) \lambda+a c d=0
$$

$$
\begin{gathered}
\Rightarrow(\gamma+\lambda+\psi-\gamma \psi)(\lambda+\mu)(\lambda+\epsilon)-((\gamma+\lambda+\psi-\gamma \psi)(\lambda+\mu)-(\gamma+\lambda+\psi-\gamma \psi)(\lambda+\epsilon)+(\lambda+\mu)(\lambda+\epsilon))- \\
((\gamma+\lambda+\psi-\gamma \psi)+(\lambda+\mu)-(\lambda+\epsilon))=0
\end{gathered}
$$

To simplify the notation, we let $B 1=(\gamma+\lambda+\psi-$ $\gamma \psi), B 2=\lambda+\mu, B 3=\lambda+\epsilon \quad$ and $\quad k=(\gamma+\lambda+\psi-$ $\gamma \psi)(\lambda+\mu)(\lambda+\epsilon)$

This implies

$$
\begin{gathered}
(\lambda+B 1)(\lambda+B 2)(\lambda+B 3)-K=0 \\
\lambda^{3}+A 1 \lambda^{2}+A 2 \lambda+A 3=0
\end{gathered}
$$

Where

$$
A 1=B 3+B 2+B 1
$$

$$
\begin{gathered}
A 2=B 3(B 2+B 1)+B 2 B 1-1 \\
A 3=B 3 B 2 B 1-K
\end{gathered}
$$

The expression for $R_{0}$ can be in terms of $B_{i}$ as

$$
R_{0}{ }^{2}=\frac{(1-\gamma) \beta}{B 3 B 2 B 1^{2}}
$$

Using the Routh-Hurwitz criterion is a method for determining whether a linear system is stable or be examining the locations of the characteristic equation of the system. In fact, the method determines only if there 
are roots that put outside of the left half plane; it does not actually compute the roots Routh-Hurwitz criteria [33].

\subsubsection{Existence of Backward Bifurcation}

We intend to determine the stability of the endemic equilibrium and to carry out the possibility of the existence of backward bifurcation due to existence of multiple equilibrium and reinfection. As a disease attacks it reduces the number of susceptible individuals in the population, which tends to reduce its reproductive rate [34]. For a backward bifurcation to occur, this means that when $R_{0}<$ 0 the endemic equilibrium point can exist as well as when $R_{0}>1$. we would expect the disease to be able to attack at $R_{0}=1$ in the case of a backward bifurcation with the properties of unstable equilibrium bifurcating from the disease-free equilibrium when $R_{0}<1$, giving rise to multiple stable states. But not in the case of a forward bifurcation, in which in the absence of a low-level unstable equilibrium when $R_{0}<1$ and a stable equilibrium bifurcating from the disease-free equilibrium when $R_{0}>1$ arise naturally when the disease does not attack when $R_{0}=1$.

\section{Methodology}

We used the number of cases reported from March 20 to May 23, 2020 to estimate the possible spread size and peak time of COVID-19, especially in Ethiopia. The basic deterministic mathematical model, SEIR model and by considering awareness compartment model, self-quarantine, infected isolated sever case, and Infected dingoes were adopted for prediction. Given that different model inputs may infer different model outputs, we implemented media effects on COVID 19 model predictions with in different groups.

\section{Simulation and Discussion}

In this section, we consider the simulation study of the system of differential equations given in (1). As stated earlier these equations describe the dynamics of human populations of the COVID 19 model that includes the effect of awareness programs. The simulation study performed using ode45 solver of MATLAB software. The RungeKutta fourth-order method conducted based on a variable step-size used for the purpose. The parametric values collected from the literature and used here. Those were not available were not obtained from literatures published by researchers in malaria endemic countries which have similar environmental conditions of our country Ethiopian current COVID 19 pandemic diseases conformed cases and death rates. We present the numerical analysis of the model (1). Those were not available were not obtained from literatures published by researchers in COVID 19 endemic countries which have similar environmental conditions. We present the numerical analysis of the model (1) and (2). The initial conditions used were form EPHI up to August 03 /2020 reported data [24].

$$
\begin{gathered}
S(0)=1000,000, E(0)=33,385 \\
S_{q}(0)=319,000, I_{a}(0)=1,311, I_{s}(0)=65,
\end{gathered}
$$

$I_{D}(0)=54,980, R(0)=7,911$. Moreover, to check the feasibility of our analysis regarding stability conditions, we have conducted some numerical computation using MATLAB by choosing the following set of parameter values in model system (1).

$$
\begin{array}{lllll}
\Psi=500 & \beta=0.0004 & q=0.0007 & q 1=0.0003 & \epsilon=0.3 \\
\lambda=0.006 & \omega=0.002 & \gamma=0.001 & \psi=0.2 & \mu 1=0.0023
\end{array}
$$

We simulate the basic COVID 19 model in find out the effects of varying each intervention parameter [20] and [2526]. Figure 2 a phase portrait illustrating the changes in the seven state variables of the assumed COVID 19 model showing the system with time, of susceptible, self-quarantine, exposed humans, infected awarded humans, isolate infected sever humans, infected diagnosed humans and shows the system of recovered humans or removed. The variations of infective $I_{s}(t), I_{D}(t)$ and $I_{a}(t)$ with respect to time ' $t$ ' for different values of the transmission rate of ${ }^{\prime} \gamma$ ' and ' $\omega$ ' shown in Figures 3, 4 and 5 respectively. From these figures, it noted that as the transmission rate of ${ }^{\prime} \gamma^{\prime}$ increases the number of recovered humans $R(t)$ and awareness programs both decreases the infected humans sever infected and infected diagnosed humans increased in number. Further, the variations of the infective population $I_{s}(t)$ and the aware population $I_{a}(t)$ with respect to time ' $t$ ' for different values of rate of transmission of awareness programs or the awarded cure or died ' $\omega$ ' were show in Figures 3, 4 and 5 respectively. From these figures, it is apparent that as the rate of transmission of awareness programs ' $\omega$ ' increases the infective population $I_{s}(t)$ decreases whereas the aware population $I_{a}(t)$ increases. In addition, from Figure 5 it is interesting to observe that for $\gamma=0$ the aware population $I_{s}(t)$ and $I_{D}(t)$ approaches 0.

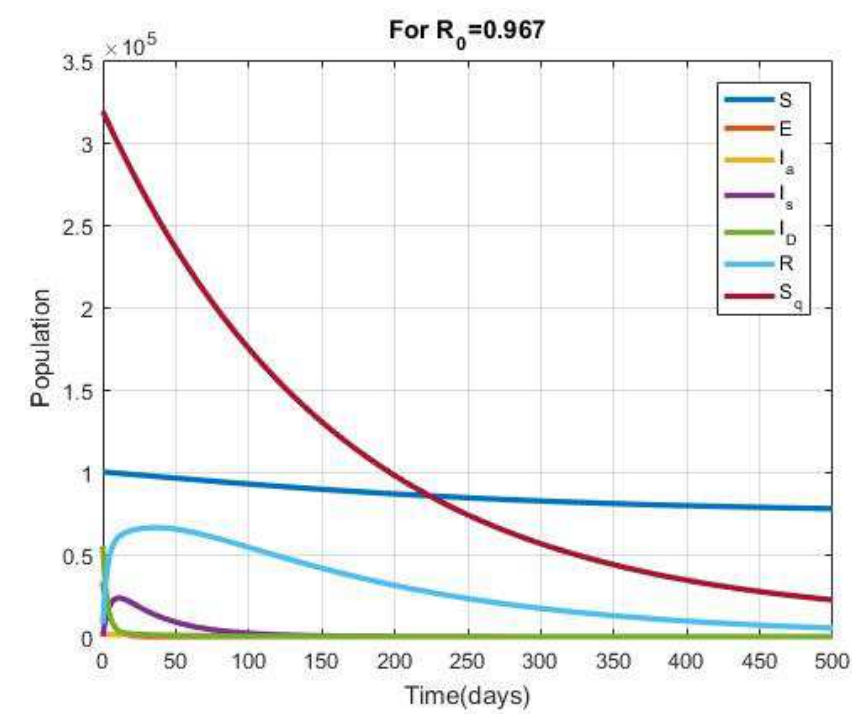

Figure 2. A phase portrait illustrating the changes in the seven state variables. 


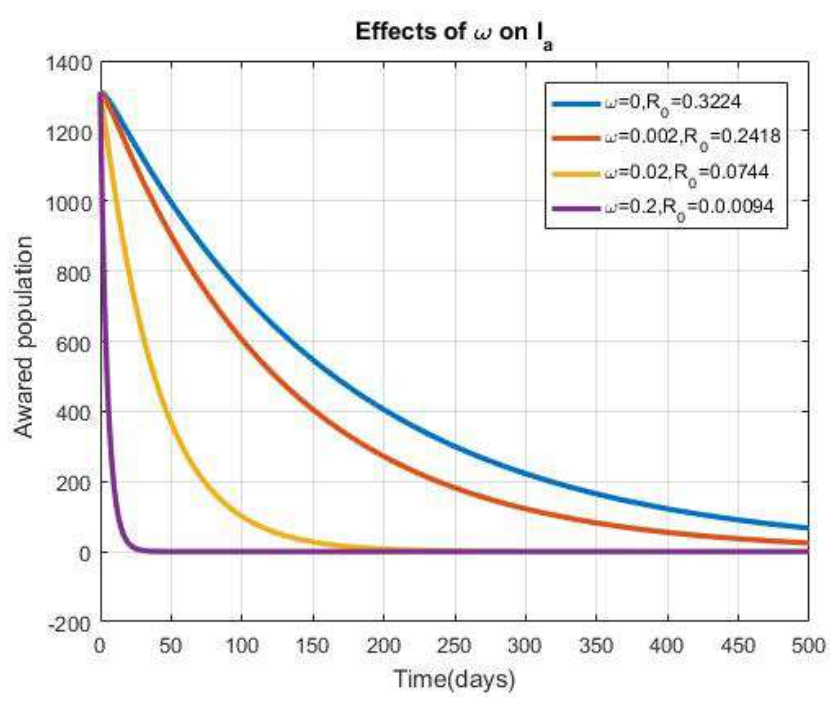

Figure 3. Variation of awarded infective population with time for different values of $\omega$.

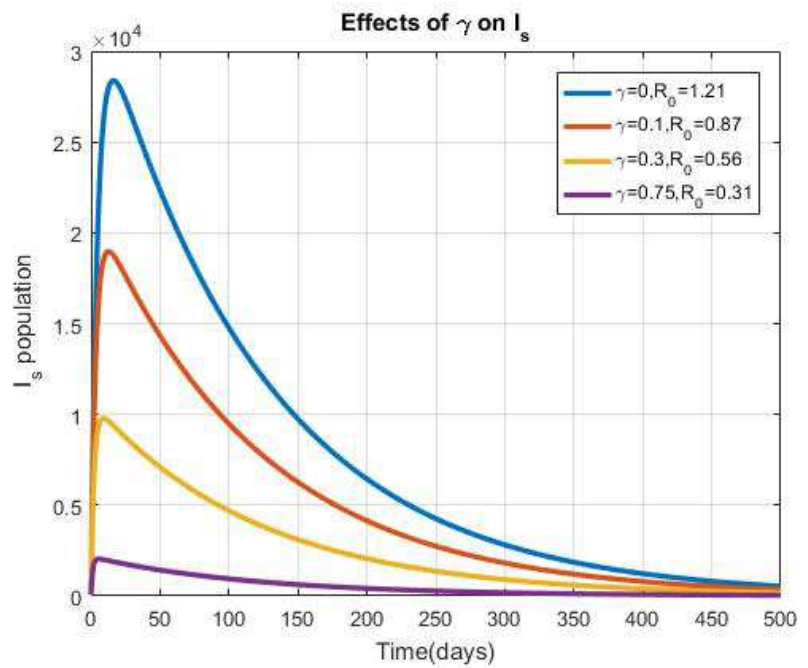

Figure 4. Variation of sever infective population with time for different values of $\gamma$.

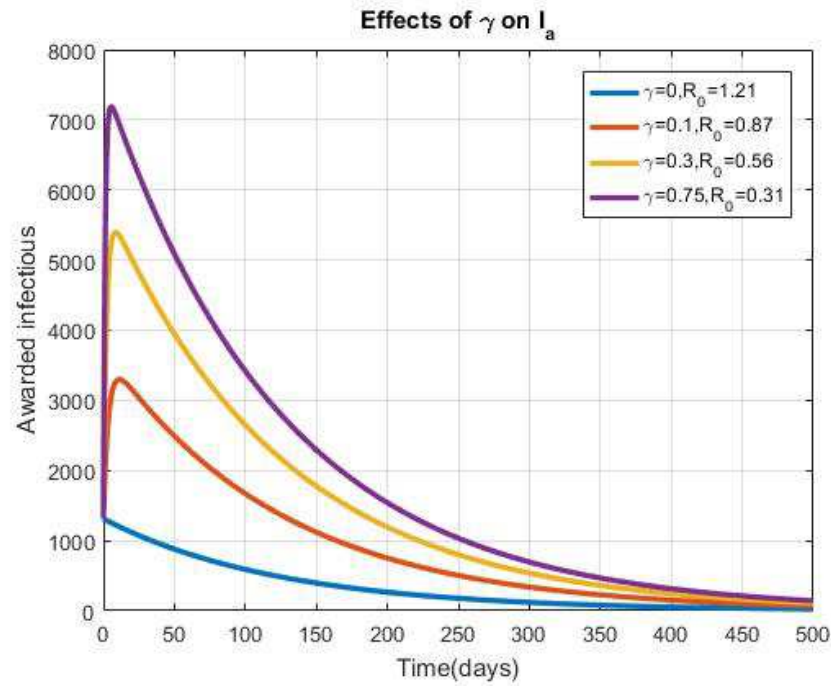

Figure 5. Variation of awarded infective population with time for different values of $\gamma$.

\section{Conclusion}

We derived and analyzed a basic deterministic mathematical model to better understand the a mathematical model to control the spread of COVID 19 Pandemic diseases mathematical model has been proposed and analyzed to study the consequences of awareness programs generated by the media in a variable population with different governmental departments. It considered that the growth rate of cumulative density of awareness programs is proportional to the number of infective, isolated infective sever and infected diagnosed. It has been assumed further that awareness causes some susceptible to isolate or self-quarantined themselves from infective forming a separate subclass in the population. The model has shown that the disease-free equilibrium is stable until, the basic reproduction number $R_{0}<0$. The disease-free equilibrium becomes unstable for $R_{0}>0$, which leads to the existence of an endemic equilibrium. The analysis demonstrates that an endemic equilibrium is locally as well as non-linearly stable under certain conditions. The model analysis further shows that awareness programs through the media campaigning are helpful in decreasing the spread of COVID 19 Pandemic diseases by isolating a fraction of susceptible from infective.

\section{Acknowledgements}

First, I would like to say thanks to my God who handed to me his peace. Next, I would like to express my sincere gratitude and appreciation goes to my former M.Sc. adviser Dr. Purnachandra Rao Koya (Prof.) for his commitment and fruitful to support me to do this work.

\section{References}

[1] Coronavirus 2019-nCoV, CSSE. Coronavirus 2019-nCoV Global Cases by Johns Hopkins CSSE (Available from: https://gisanddata.maps.arcgis.com/apps/opsdashboard/index.

[2] Tyrrell DA, Bynoe MLn (1966). Cultivation of viruses from a high proportion of patients with colds. Lancet: 1: 76-77.

[3] GISAID Global Initiative on Sharing All Influenza Data Phylogeny of SARS-like beta coronaviruses including novel coronavirus (nCoV). (Available from: https://nextstrain.

[4] Zhou P, Yang XL, Wang XG et al (2020). A pneumonia outbreak associated with a new coronavirus of probable bat origin. Nature https://doi.org/10.1038/s41586-020-2012-7

[5] Rottier PJM. The Coronaviridae Siddell SG, editor. 115-1372013. Springer Science \& Business Media. (Available from: https://link.springer.com/content/pdf/10.1007\%2F978-1-4899 1531-3_6.pdf).

[6] Chan JF, Yuan S, Kok KH et al (2020) A familial cluster of pneumonia associated with the 2019 novel coronavirus indicating person-to-person transmission: a study of a family $\begin{array}{llll}\text { cluster Lancet. S0140-6736 } & \text { (20) 30154- }\end{array}$ https://doi.org/10.1016/S0140-6736(20)30154-9

[7] Li Q, Guan X, Wu P et al (2020) Early transmission dynamics in Wuhan, China, of novel coronavirus-infected pneumonia. N Engl J Med. https://doi.org/10.1056/NEJMoa2001316 
[8] Guan W, Ni Z, Yu H, et al (2020) Clinical characteristics of 2019 novel coronavirus infection in China, medRxiv preprint posted online on Feb. 9, https://doi.org/10.1101/2020. 02.06.20020974.

[9] WHO (2020) COVID-19 data accessed April 23.

[10] African CDC (2020) https://africacdc.org/covid-19/ accessed April 2.

[11] WHO (2020) EMRO http://www.emro.who.int/index.html accessed April 6.

[12] IOM (2020) east and horn of Africa regional strategic preparedness and response plan covid-19, updated on 6 April 2020.04.09.20059154v1.

[13] FMOH (2020) national comprehensive covid-19 management handbook Ethiopia First edition May 23.

[14] Ferguson N. M, Laydon. D, Nedjati-Gilani. G, Imai. N, Ainslie. K, Baguelin. M, Bhatia S, Boonyasiri A et al (2020) Impact of non-pharmaceutical interventions (NPIs) to reduce COVID-19 mortality and healthcare demand. London: Imperial College COVID-19 Response Team, March 16.

[15] Murray. C. JL, IHME (2020) COVID-19 health service utilization forecasting team. Forecasting COVID-19 impact on hospital bed-days, ICU-days, ventilator-days and deaths by US state in the next 4 months 2020.03.27.20043752; https://doi.org/10.1101/2020.03.27.20043752

[16] WHO (2020) Coronavirus disease (COVID2019) situation reporthttps://www.who.int/docs/defaultsource/coronaviruses/si tuati reports/20200219 sitrep30 covid19 accessed March 31.

[17] Martinez-Alvarez. M, Jarde. A, Usuf. E, Brotherton. H, Bittaye. M, Samateh Al, Antonio. Met. Al (2020) COVID-19 pandemic in West Africa Lancet Glob Health 2020 April 1.

[18] Zou L Ruan F, Huang M, Liang L, Huang H, Hong Z, Yu J, et. al (2020), SARSCoV2 viral load in upper respiratory specimens of infected patients. N Engl J Med; 382: 11771179.

[19] Kinfu. Y, Opiyo. C, \& Wamukoya, M. (2014). Child Health and Mortality in sub-Saharan Africa: Trends, causes, and forecasts. In C. O. Odimegwu, \& J. Kekovole (Eds.), Continuity and change in sub-saharan African Demography (Vol. 17, pp. 60-77) (Routledge African Studies; Vol. 17). New York USA: Routledge https://doi.org/10.4324/9781315879444

[20] Constantine's I Siettos1, and Lucia Russo2 (2013;) Mathematical modeling and research of infectious disease dynamics Virulence 4: 4, 295-306; May 15, (C) 2013 Landes Bioscience.

[21] O. Diekmann, J. A. P. Heesterbeek and J. A. J. Metz (1990), on the definition and computation of the basic reproduction ratio in models for infectious diseases in heterogeneous populations, J. Math. Biol, 28 365-382, doi: 10.1007/BF00178324.156.

[22] K. Dietz (1993), "The Estimation of the Basic Reproduction Number for Infectious Diseases", Statistical Methods in Medical Research, Vol. 2, Pp. 23-41.

[23] Li Y, Wang B, Peng R, Zhou C, Zhan Y, Liu Z, et al (2020)
Mathematical Modeling and Epidemic Prediction of COVID19 and Its Significance to Epidemic Prevention and Control Measures Ann Infect Dis Epidemiology; 5 (1): 1052. ISSN: 2475-5664.

[24] EPHI (2020) National public health emergency operation (PHEOC), COVID 19 pandemic preparedness and response in Ethiopia, August 03-augst 09, 2020. www.ephi.gov.et, N NA.

[25] A. K. Misraa, Anupama Sharmaa, J. B. Shuklab (2011), Modeling and analysis of effects of awareness programs by media on the spread of infectious diseases Mathematical and Computer Modelling 53 1221-1228 doi: 10.1016/j.mcm.2010.12.005.

[26] Fekadu Tadege Kobe, Purnachandra Rao Koya. Controlling the Spread of Malaria Disease Using Intervention Strategies. Journal of Multidisciplinary Engineering Science and Technology (JMEST). Vol. 2, Issue 5, May 2015, pp 1068 74. ISSN: $3159-0040$. http://www.jmest.org/wpcontent/uploads/JMESTN42350745.pdf

[27] Geremew Kenassa Edessa, Purnachandra Rao Koya.

Modeling and Stability Analysis of Host - Parasite Population Dynamics. Mathematical Modelling and Applications. Vol. 5, No. 2, 2020, pp 28 doi 10.11648/j.mma.20200502.17 http://www.sciencepublishinggroup.com/journal/paperinfo?jo urnalid $=389 \&$ doi $=10.11648 /$ j.mma.20200502.17

[28] Dereje Gutema Edossa, Alemu Geleta Wedajo, Purnachandra Rao Koya. Modeling the Dynamics of Endemic Malaria Transmission with the Effects of Control Measure. American Journal of Applied Mathematics. Vol. 8, No. 3, 2020, pp. 158170. Doi: 10.11648/j.ajam.20200803.17.

[29] Carlos Castillo-Chavez, Wenzhang Huang, Jia Li, Competitive exclusion and coexistence of multiple strains in an SIS STD model, SIAM Journal on Applied Mathematics 59 (5) (1999) $1790-1811$.

[30] Birkhoff, G and Rota, G. C (1982), Ordinary Differential Equations, Ginn.

[31] Alemu Geleta Wedajo, Boka Kumsa Bole, Purnachandra Rao Koya. The Impact of Susceptible human Immigrants on the Spread and Dynamics of Malaria Transmission. American Journal of Applied Mathematics. Vol. 6, No. 3, 2018, pp. 117 127. Doi $\quad 10.11648 /$ j.ajam.20180603.13 http://article.sciencepublishinggroup.com/pdf/10.11648.j.ajam .20180603.13.pdf

[32] Van den Driessche, P. and Watmough, J. (2002). Reproduction numbers and sub threshold endemic equilibria for compartmental models of diseastransmission. Mathematical Biosciences, $180, \quad 29$ http://dx.doi.org/10.4236/health.2014.610120.

[33] Flores J. D. (2011). Math-735: Mathematical Modelling, Routh-Hurwitz criteria. Department of Mathematical Sciences, The University of South Dakota, jflores@usd.edu.

[34] Eshetu Dadi Gurmu, Boka Kumsa, Purnachandra Rao Koya. Optimal control Strategy on Human Papilloma Virus (HPV) model with Backward Bifurcation Analysis. IOSR Journal of Mathematics (IOSR-JM), Vol. 15, Issue 6, Ser. II (Nov - Dec 2019), Pp 65-87. DOI: 10.9790/5728-1506026587 http://www.iosrjournals.org/iosr-jm/papers/Vol15issue6/Series-2/G1506026587.pdf 